\title{
Migrantische Netzwerke und Integration: \\ Das transnationale Kommunikationsfeld deutscher Einwandererfamilien in den USA
}

\author{
Félix Krawatzek • Gwendolyn Sasse
}

Online publiziert: 18. Januar 2018

(C) Der/die Autor(en) 2018. Dieser Artikel ist eine Open-Access-Publikation.

Zusammenfassung Basierend auf einer Sammlung von 1000 Briefen deutscher Auswanderer in den USA analysiert dieser Aufsatz die Entwicklung und Bedeutung transatlantischer und deutsch-amerikanischer migrantischer Netzwerke über einen Zeitraum von 150 Jahren. Wir unterscheiden zwischen „starken“ und „schwachen“ Beziehungen, die diesen Netzwerken zugrunde liegen, und untersuchen die politischen Faktoren, die die verschiedenen Formen von Netzwerken prägen. Unsere Studie konzentriert sich auf die Schnittstellen zwischen den transatlantischen und deutsch-amerikanischen migrantischen Netzwerken. Sie kontrastiert dabei Elemente des 19. Jahrhunderts, ein Zeitalter dichter migrantischer Netzwerke, die sich weitestgehend ohne staatliche Einwirkung entfalteten, mit der schrittweisen Auflösung und geringeren Verbindlichkeit deutsch-amerikanischer und transatlantischer Netzwerke im 20. Jahrhundert. Die Bandbreite der bisher nur punktuell erforschten Auswandererbriefe veranschaulicht sowohl die Diversität von Integrationserfahrungen als auch die Gleichzeitigkeit von kultureller Anbindung ans Heimatland und wirtschaftlicher und gesellschaftlicher Integration in den USA über einen langen Zeitraum hinweg.

Schlüsselwörter Transnationalismus · Integration · Auswanderung · Migrantische Netzwerke · Auswandererbriefe

Dr. F. Krawatzek $(\bowtie) \cdot$ Prof. G. Sasse

Department of Politics and International Relations, Nuffield College, University of Oxford, New Road, Oxford, OX1 1NF, Großbritannien

E-Mail: felix.krawatzek@politics.ox.ac.uk

Prof. G. Sasse

E-Mail: gwendolyn.sasse@ politics.ox.ac.uk 


\title{
Migrant networks and integration: The transatlantic field of communication of German immigrant families in the US
}

\begin{abstract}
Drawing on a collection of 1000 letters sent by German migrants in the US we analyse the development and relevance of transatlantic and GermanAmerican migrant networks over a period of 150 years. We distinguish between "strong" and "weak" ties which are at the heart of these networks and identify the political factors that influence the different types of networks. Our study focuses on the connections between transatlantic and German-American migrant networks. We contrast elements of the 19th century, a period of dense migrant networks which developed largely without state interference, with the gradual weakening of both types of networks in the 20th century. The empirical detail contained in the as yet underexplored historical letter collection emphasises both the diversity of integration experiences and the simultaneity of a cultural attachment to the country of origin and economic and social integration in the country of residence over a long period of time.
\end{abstract}

Keywords Transnationalism · Integration · Emigration · Migrant networks · Migrant letters

\section{Einleitung ${ }^{1}$}

Historische Fallstudien bereichern sozialwissenschaftliche Analysen, wenn sie den zeitlichen Horizont von Konzepten und Empirie erweitern und somit die Dynamiken von Kontinuität und Wandel differenzieren. Green und Waldinger haben zu Recht dazu aufgerufen, weniger auf die Frage zu konzentrieren, wie „neu“ das Phänomen Transnationalismus ist, und vielmehr längerfristigen Entwicklungen systematisch nachzugehen (Green und Waldinger 2016, S. 1). ${ }^{2}$ Somit werden die Stabilität, Intensität und Bedeutung von grenzübergreifenden migrantischen Tätigkeiten selbst zum Forschungsobjekt gemacht. Hier knüpft dieser Aufsatz an, der sich am Beispiel der Einwanderung der Deutschen in die USA im 19. und 20. Jahrhundert einem Schlüsselbegriff der Migrationsforschung - migrantischen Netzwerken - widmet. Anhand von Textlängsschnittdaten - etwa 1000 zwischen den Migranten und Migrantinnen und deren Familien und Bekannten zirkulierenden Briefen - untersuchen wir im diachronen Vergleich von drei durch politische Krisenmomente voneinander getrennten Zeitabschnitten die Entwicklung sowohl transatlantischer als auch deutsch-amerikanischer migrantischer Netzwerke.

\footnotetext{
1 Die diesem Artikel zu Grunde liegende Forschung ist durch das vom Leverhulme Trust geförderte Projekt „Political Remittances: Understanding the Political Impacts of Migration“ (M3RVQL00) und einen Small Award des John Fell Fund/OUP Research Fund (143/003) ermöglicht worden. Der Autor und die Autorin danken Christian Fastenrath für seine Unterstützung bei der Aufbereitung der Textdaten.

2 Eine solche diachrone Vorgehensweise zur Schärfung des Begriffs „Transnationalismus“ findet sich bspw. auch in (Smith 2000).
} 
Der Begriff „migrantische Netzwerke“ ist dabei bewusst gewählt, um die in der Migrationsforschung vorgenommene Unterscheidung zwischen „Migrationsnetzwerken“, die den Migrationsprozess ermöglichen und begleiten, und „Migrantennetzwerken" unter Einwanderern im Ankunftsland, nicht als Grundannahme zu übernehmen, sondern zum Teil der Forschungsfrage zu machen. Bestehende Kategorisierungen suggerieren klare Unterscheidungen von Aspekten die im migrantischen Alltag miteinander verwoben sind. Es sind vielmehr die Schnittstellen zwischen den verschiedenen Netzwerken, die Aufschlüsse über den Wandel und die Kontinuität von Migrationserfahrungen ermöglichen.

Darüber hinaus liegt der Fokus in der Migrationsforschung auf den Auswirkungen von Netzwerken, die implizit als stabile Variable konzipiert sind. Die Entstehung und beständige Weiterentwicklung verschiedener Netzwerke über einen längeren Zeitraum hinweg sind bisher weniger ins Blickfeld gerückt worden. Dieser Aufsatz zeichnet hingegen die Entwicklung migrantischer Netzwerke aus der Perspektive der Briefeschreiber nach.

Durch wirtschaftliche und zum Teil politische Ursachen motiviert, wuchs die deutsche Einwanderung in die USA ab etwa $1820 \mathrm{zu}$ einer anhaltenden Massenmigration (Helbich 1988; Roeber 1997). Insbesondere im 19. Jahrhundert war die „deutsche“ Kultur ein sichtbares Element des öffentlichen Lebens in den USA (Bergquist 1984, S. 9): deutsche Siedlungen, Schulen, Vereine und Kirchen waren bis zum Ersten Weltkrieg ein fester Bestandteil des migrantischen Alltags (Hawgood 1940; Kazal 2004). Das weit gefächerte und häufig auf die USA übertragbare berufliche Profil deutscher Auswanderer trug dazu bei, dass sich im Gastland eine fast alle Lebensbereiche umfassende ,deutsche“ Infrastruktur etablieren konnte. Die sich daraus ergebenden Netzwerke leisteten praktische und emotionale Hilfestellung, unterstützen Ausreisewillige aus verschiedenen Ländern (Guillet 1963) und schufen Räume für den Austausch über das Ankunfts- und Heimatland ${ }^{3}$.

Die deutschsprachigen Auswanderer werden generell als schnell assimilierte Gruppe wahrgenommen. Erst um die Jahrhundertwende, in Zeiten stark zunehmender Auswanderung aus Süd- und Osteuropa, setzte man sich in den USA mit Einwanderung als einem politischen Problem tiefergehend auseinander. Präsident Roosevelt beauftragte Senator Dillingham 1907 damit, die Lebenssituation der verschiedenen Einwanderergruppen durch eine Einwanderungskommission zu erforschen (Immigration Commission and Dillingham 1911). Die deutschsprachigen Einwanderer wurden bereits zu diesem Zeitpunkt als weitestgehend integrierte Gruppe dargestellt, deren Sichtbarkeit in Folge des Ersten Weltkriegs weiter abnahm (Conzen 1980, S. 406). Diese Assimilation trotz der Größe der „Migrantengruppe“ und ihren weitläufigen organisatorischen Strukturen macht sie zu einem relevanten Fallbeispiel. Wenn unsere Analyse zeigt, dass selbst der Alltag dieser Migranten und Migrantinnen über einen längeren Zeitraum hinweg von Transnationalität

\footnotetext{
${ }^{3}$ Die Begriffe „Gastland“ und „Ankunftsland“ bzw. „Heimatland“, „Herkunftsland“ und „Ursprungsland“ werden hier weitgehend als Synonyme variiert, die im jeweiligen Kontext eine gewisse Schwerpunktsetzung erlauben. Keiner der Begriffe bietet jedoch die nötige begriffliche Trennschärfe - insbesondere der Begriff „Heimatland“ und generell die Fokussierung auf einzelne Länder als Bezugspunkte bleiben problematisch. Wie variieren die Begriffe „Auswanderer“ und „Einwanderer“ je nach Bezug auf Herkunftsort und Migrationsprozess bzw. das Leben in den USA.
} 
geprägt blieb, ließe dies auf ein ähnliches oder größeres $\mathrm{Maß}$ an transnationalen Verflechtungen bei anderen, weniger schnell assimilierten Einwanderergruppen schließen.

Die erste Generation der Einwanderer in den USA pflegte enge Verbindungen zu ihren Familien und Bekannten in Deutschland, doch über einen längeren Zeitraum betrachtet sind die Effekte verschiedener politischer Kontexte für die Evolution der migrantischen Netzwerke und der gelebten Transnationalität bedeutsamer als die Unterscheidung zwischen Generationen. Das transnationale Kommunikationsfeld prägte das überwiegend positive Amerikabild in Deutschland nachhaltig. V. a. die Briefe aus dem 19. Jahrhundert betonen die eklatante Kluft zwischen beiden Ländern im Hinblick auf Modernisierungsprozesse und damit verbundene wirtschaftliche Möglichkeiten und politische Freiheiten. Auch wenn es bereits früh warnende Stimmen unter den Auswanderern gab, z. B. Klagen über wirtschaftliche Risiken und die Gefahren der Mechanisierung der Gesellschaft, so kippte die Stimmung erst im Kontext der deutschfeindlichen Propaganda vor und während des Ersten Weltkriegs. ${ }^{4}$ Das in der Zeit der Weimarer Republik in die alte Heimat „versandte“ Amerikabild war somit vielschichtiger: Das Gefühl, in den USA nicht mehr willkommen zu sein, führte zu einer teilweisen Distanzierung. Davon zeugten auch die Berichte zeitgenössischer amerikanischer Soziologen (Kellor 1920, S. 25).

Unsere Analyse differenziert zwischen den transnationalen Netzwerken der Auswanderer und den deutsch-amerikanischen Netzwerken in den USA. In Bezug auf unsere Fallstudie interessieren uns die gruppeninterne und kontextabhängige Diversität der Identitäten und Integrationserfahrungen (Wimmer and Glick Schiller 2002). Unsere Daten decken sowohl Zeiten der nur ansatzweise vom amerikanischen Staat regulierten Einwanderung, des einfachen Erwerbs der amerikanischen Staatsbürgerschaft und der Vielfalt an ,ethnisch“ oder sprachlich definierten Organisationen im 19. Jahrhundert ab, als auch eine zunehmend restriktive Politik Anfang des 20. Jahrhunderts, einschließlich gezielter Maßnahmen gegen die Deutschen als potenzielle Verräter während des Ersten Weltkriegs.

Unsere Studie beginnt mit der Idee, dass „Assimilation und anhaltende transnationale Verbindungen weder inkompatibel noch binäre Gegensätze sind“ (Levitt and Glick Schiller 2004, S. 1003). Die Linearität von Assimilation oder Integration wird seit längerem empirisch und konzeptuell in Frage gestellt (Freeman 2004), doch es bleibt eine Herausforderung, die Gleichzeitigkeit von Integration und Transnationalität systematisch über einen längeren Zeitraum hinweg empirisch zu erfassen. Die historische Perspektive vermittelt uns hierbei einen Einblick in die Rolle von migrantischen Netzwerken im transnationalen Alltag (siehe z. B. die soziologischen Studien von Gans zu den Italienern in West-Boston (1962) oder Kornblums Studie über die Arbeiter-Community in Chicago (1974)).

\footnotetext{
${ }^{4}$ Einen guten Einblick in die Ambivalenz des deutschen Amerikabildes gewährt die zeitgenössische Literatur, siehe (Mikoletzky 1988).
} 


\section{Migrantische Netzwerke und Transnationalismus}

In der Migrationsforschung wird zwischen Migrantennetzwerken (Bommes 2011) und Migrationsnetzwerken (Bade 2003; Haug 2008), unterschieden. Die Netzwerke selbst und ihr soziales Umfeld sind jedoch selten der Hauptforschungsgegenstand. In empirischen Studien werden verschiedene migrantische Netzwerke meist als unabhängige Variablen konzeptualisiert, die zur Erklärung beispielsweise für den Grad an Integration im Ankunftsland (Poros 2001), für das Entwicklungspotenzial des Herkunftslandes (Lacroix 2009) oder für das Zusammengehörigkeitsgefühl und politische und soziale Engagement im Heimatland (Ahmadov and Sasse 2015; Burgess 2012,) herangezogen werden.

Im Unterschied zum bisherigen sozialwissenschaftlichen Forschungsschwerpunkt richten wir unser Augenmerk auf migrantische Netzwerke, bewusst allgemeiner formuliert, als abhängige Variable und versuchen, die Entstehung verschiedener Netzwerke und die Veränderungen in ihrer Zusammensetzung und Funktion nachzuvollziehen. Dabei geht es uns darum zu verstehen, wie die Auswanderer verschiedene Netzwerke in den Briefen thematisierten, als maßgeblich für ihren Alltag darstellten und dadurch zu einer Realität im transatlantischen Kommunikationsfeld werden ließen. Unsere qualitative Textanalyse hebt die Bedeutung der politischen Beziehungen zwischen ,neuer“ und ,alter“ Heimat für die an migrantische Netzwerke geknüpften Erwartungen und Erfahrungen hervor. Drei durch politische Krisen voneinander getrennte Zeitabschnitte markieren Verschiebungen an der Schnittstelle zwischen transatlantischen und deutsch-amerikanischen Netzwerken. Jede dieser Verschiebungen reaktiviert die Gleichzeitigkeit von Integration und Transnationalität: in der Frühphase (bis zum Ersten Weltkrieg) bedingen sich transatlantische und deutschamerikanische Netzwerke gegenseitig, und transatlantische Netzwerke gehen teilweise in deutsch-amerikanischen Netzwerken auf; in der Zwischenkriegszeit werden transatlantische Netzwerke wiederbelebt während deutsch-amerikanische Netzwerke abschwächen, und in der Zeit nach dem Zweiten Weltkrieg verlieren nach den deutsch-amerikanischen Netzwerken auch die verbliebenen transnationalen Netzwerke an Bedeutung.

Über die eigene Beziehung zum Ankunfts- und Herkunftsland zu schreiben, ist ein Schritt, eine Identität zu (re-)aktivieren. Green und Waldinger haben auf die Notwendigkeit hingewiesen, bei der Analyse transnationaler Verflechtungen über migrantische Aktivitäten hinaus zu schauen und die Rolle der Herkunfts- und Gastländer und ihr Verhältnis zueinander stärker ins Visier zu nehmen (2016, S. 3). Wir leiten daraus unsere Hypothese ab, dass politische Krisen im Verhältnis zwischen Ankunfts- und Herkunftsland migrantische Netzwerke stärkt bzw. schwächt und damit die Gewichtung in der gleichzeitigen Identifizierung mit „hier“ und „draußen“ (in den Worten der Briefeschreiber) verändern.

Die Relevanz von Granovetters These von der Stärke schwacher Beziehungen (1973), die für Kommunikation und soziale Mobilität angeblich wichtiger sind als starke, transitive Beziehungen im Familien- und Freundeskreis, ist in der Migrationsforschung immer wieder betont worden. Hierbei erschweren jedoch die sehr unterschiedlichen Definitionen von „schwachen Beziehungen“ - von Akteuren mit einer Vermittlerrolle auf dem Arbeitsmarkt (Elrick and Lewandowska 2008) bis hin 
zum weiteren Familien- und Bekanntenkreis (Liu 2013) - die Interpretation. Zunächst wurden Migrationsnetzwerke, definiert als die sozialen Bindungen zwischen Migrantengruppen im Herkunfts- und Zielland, hauptsächlich als der Faktor gesehen, der Migrationsbewegungen bedingt. Netzwerke transportieren Informationen sowie soziale und finanzielle Unterstützung (Massey and Espana 1987) und wurden ursprünglich hauptsächlich in eine Richtung - adressiert an potenzielle Auswanderer - gedacht.

Auch die finanziellen, sozialen und politischen Transfers (remittances) von Migranten und Migrantinnen zurück in ihr Heimatland setzen die Existenz von transnationalen Netzwerken voraus. Die vom Konzept des Transnationalismus geleitete sozialwissenschaftliche Forschung hat sich hier vor allem auf das Entwicklungspotenzial der Transfers und die Organisationen, durch die sie vermittelt werden, konzentriert (Glick Schiller and Fouron 1999; Levitt 2001).

Ende der 80er-Jahre rief Boyd dazu auf, der Frage nachzugehen, unter welchen Bedingungen sich Netzwerke (nicht) herausbilden und stärker bzw. schwächer werden (1989, S. 654-55). Neben geschlechtsspezifischen Aspekten betonte sie, dass die sozialen Netzwerke von Migranten und Migrantinnen zeit- und raumübergreifend sind (ibid.: 639) - dieser Aspekt wurde bisher kaum aufgegriffen. Herz und Olivier haben darauf verwiesen, dass die Netzwerkterminologie häufig lediglich als Metapher fungiert (2012, S. 115). Der Mangel an Forschung zu den Konturen migrantischer Netzwerke liegt auch in der Schwierigkeit begründet, die verschiedenen, sich überlappenden und verändernden Netzwerke einer oder mehrere Personen zu erfassen, gleich welcher Methodik man sich bedient.

Ein Eckpfeiler in der historischen Forschung zu migrantischen Netzwerken ist die soziologische Arbeit von Thomas und Znaniecki, die sich bereits vor über 100 Jahren der Erfahrung der ländlichen polnischen Immigration in die USA widmete. Aus einer Vielzahl von Briefen konstruierten sie ein Metanarrativ und argumentierten, dass im Laufe des 19. Jahrhunderts eine spezifische polnisch-amerikanische Gesellschaft entstand, die für die Integration von Neuankömmlingen in den USA ausschlaggebend war (1927). Dieser vielzitierte und dennoch ,,vernachlässigte Klassiker“ (Bulmer 1983, S. 474) nutzt verschiedene autobiographische Dokumente unter anderem um den Auswirkungen von Industrialisierung auf das Selbstverständnis polnischer Bauern und deren Gruppenzugehörigkeit nachzugehen. Dabei wird ihre Entscheidung auszuwandern im Kontext der sozialen Verschiebungen im Heimatland verortet. Unsere Analyse setzt hingegen mit dem Beginn des Migrationsprozesses an.

Die durch Briefe kommunizierten Ideen über die politische, wirtschaftliche und soziale Lage in den USA verkörperten eine konkrete Alternative aus der sich die Massenmigration speiste (Taylor 1971, S. 66). Öffentlich zugängliche Informationen für potenzielle Migranten und Migrantinnen wurden häufig im Auftrag von deutschen oder amerikanischen Ministerien verfasst. Im Vergleich dazu versprach eine als authentisch wahrgenommene private Quelle einen direkteren Zugang. Paradoxerweise waren diejenigen mit wenig Zugang zu öffentlichen Informationen und niedrigem Bildungsstand in der Praxis oft am besten über das Zielland informiert (Grabbe 1997, S. 82).

Nicht nur deutschsprachige Einwanderer erreichten die USA mit einem schwach ausgeprägten Verständnis von nationaler Identität - es dominierten familiäre, reli- 
giöse und regionale/lokale Bezugspunkte. In Anbetracht ihrer geographischen und sozialen Vielfalt definierten Auswanderer häufig erst im Zuge der Migrationserfahrung und der dafür notwendigen Hilfestellung durch migrantische Netzwerke eine Identität ,als Deutsche“, als „Iren“ (siehe Miller 2008, S. 9) usw. Durch transatlantische Kommunikationsnetzwerke erhielten die Auswanderer Informationen aus der Heimat, die es ihnen ermöglichten, sich von der dominanten Kultur sowie anderen Migrantengruppen abzugrenzen (siehe Helbich (1988) zur deutschen, Attebery zur schwedischen (2007), Liu zur chinesischen (2005), Serra zur italienischen (2009) oder Krabbendam zur niederländischen Auswanderung (2009)).

\section{Methodik und Kontext}

Die deutschsprachige Auswanderung in die USA ist eine ideale Fallstudie zur Dynamik von Kettenmigration. Die Auswanderung erfolgte über mehrere Jahrhunderte, erstreckte sich über verschiedene deutschsprachige Regionen und erfasste weite Teile der USA. Die Diversität dieser Auswanderer, ihr interner Organisationsgrad und die allmählich skeptischere politische Grundeinstellung gegenüber Immigration in den USA sowie Deutschlands Rolle in der internationalen Politik machen dieses historische Beispiel besonders relevant für ein besseres Verständnis von migrantischen Netzwerken und ihrer Bedeutung für Integration und Identitäten.

Um 1820 waren weniger als fünf Prozent aller Einwanderer in den USA als „Deutsche“ registriert. Bis 1900 stieg ihr Anteil auf etwa 25\% der gesamten außerhalb der USA geborenen Bevölkerung (insgesamt knapp über 10Mio. Menschen) (Immigration Commission and Dillingham 1911, S. 409-16). Von 1836 bis 1845 kamen jährlich ungefähr 20.000 Deutsche in den USA an; und zwischen 1852 und 1854 über eine halbe Million als die „,deutsche“ Einwanderung über die Hälfte der gesamten Immigration in die USA ausmachte. Mit insgesamt 5,5 Mio. Einwanderern von 1820 bis 1920 blieben die Deutschen die größte Einwanderergruppe (etwa 30Mio.) (Fairchild 1913, S. 189).

Auswandererbriefe sind eine anschauliche und aus sozialwissenschaftlicher Perspektive weitgehend unerforschte Quelle. Sie stellen uns jedoch vor methodologische Herausforderungen. Zum einen können wir nicht mit Sicherheit sagen, welche Auswandererfamilien Briefe geschrieben haben. Zum anderen ist eine Briefesammlung nie nach einem Zufallsprinzip erstellt worden und somit nicht automatisch repräsentativ für die Gesamtheit der geschriebenen Briefe. Zu wenig ist darüber bekannt, wie die Nachfahren einen Teil der Briefe durch eine Mischung aus bewusster Selektion und Zufall aufbewahrt haben.

Die Briefesammlung, auf die sich unsere Analyse stützt ist zumindest nicht systematisch verzerrt. Sie deckt die Hauptwellen der deutschen Auswanderung, ab und schließt deren zentrale (hauptsächlich ländliche) Gebiete ein und spiegelt sowohl die weite territoriale Ausbreitung als auch die deutschsprachigen Zentren wider (ein Großteil der Briefe stammt aus New York (Stadt und Staat), dem mittleren Westen der USA sowie Texas). Die Sammlung reicht von 1830 bis 1970 und weist eine hohe Konzentration um 1860, die Jahrhundertwende, die Zwischenkriegszeit sowie die ersten Jahre nach dem zweiten Weltkrieg auf. 
Die Briefe berichten sowohl von positiven als auch von negativen Erfahrungen. Es fehlen allerdings systematische sozioökonomische und biographische Hintergrundinformationen. Wir beschränken uns hier auf etwa 1000 Briefe der insgesamt ca. 8000 Briefe umfassenden Sammlung in der Forschungsbibliothek Gotha, für die Zusatzinformationen über das Ausreisedatum und den beruflichen Werdegang vorliegen. ${ }^{5}$ Darüber hinaus hilft die Einbindung unserer Ergebnisse in die bestehende historische Forschung. Unsere Erwartung ist nicht, dass unsere im Vergleich zu klassischen historischen Studien vom Volumen her umfassendere Quellenbasis eine Revision bisheriger Forschungsergebnisse einfordert. Es ist vielmehr deren Relevanz über einen längeren Zeitraum hinweg, die auf dem Prüfstand steht, sowie die Faktoren, die die Existenz und Ausrichtung von migrantischen Netzwerken bestimmen.

Die Briefe sind eine aussagekräftige Quelle die einen unmittelbaren Einblick in die persönlichen Erfahrungen und Ansichten von „,normalen“ Migranten und Migrantinnen vermitteln. Von Intellektuellen verfasste Darstellungen für eine größere, öffentliche Leserschaft, mögen in Bezug auf bestimmte Themen und Empfängerkreise eine besondere Bedeutung gehabt haben, aber ihre Zielsetzungen waren grundlegend andere. Die von uns genutzte Sammlung erfasst hauptsächlich Arbeitsmigration, obgleich auch als politische Flüchtlinge kategorisierte Auswanderer in den Briefen vereinzelt zu Worte kommen.

Die idiosynkratrischen Zeugnisse der häufig ungeübten Briefeschreiber haben wir mit Hilfe einer qualitativen Textanalyse erfasst. Unser in einem iterativen Verfahren entwickeltes Kodierschema ordnet die Inhalte der Briefe Kategorien, wie z.B. politischen Ereignissen, Arten von migrantischen Netzwerken oder verschiedenen Integrationsdimensionen zu, um diese in einem zweiten Schrittsystematisch auswerten zu können. Für diesen Aufsatz haben wir uns auf die Briefe konzentriert, die Einblicke in migrantische Netzwerke ermöglichen. Aus ihnen zitieren wir im folgenden empirischen Teil typische Beispiele, die größere Trends veranschaulichen sollen. ${ }^{6}$

Bei der Textanalyse unterscheiden wir zwei Arten von personenzentrierten Netzwerken: transatlantische Netzwerke und deutsch-amerikanische Netzwerke in den USA. Persönliche transatlantische Netzwerke beruhen auf dem kontinuierlichen Austausch zwischen Auswanderern und ihren Familien und Bekannten im Heimatland. Diese transnationale Kommunikation fand insbesondere im 19. Jahrhundert zwischen Gruppen (d.h. Familien, Bekanntenkreisen, ganzen Ortschaften) statt. Die daraus resultierende Reichweite der Briefe unterstreicht die Relevanz dieser Briefe

\footnotetext{
5 Das insgesamt etwa 8000 Briefe umfassende, in der Forschungsbibliothek Gotha archivierte Korpus ist in den letzten 30 Jahren mit Hilfe von Aufrufen an die Öffentlichkeit gesammelt worden. Unsere Studie ist der erste Versuch, über bestehende punktuelle Untersuchungen hinauszugehen. Wir danken Prof. Ursula Lehmkuhl für den umfassenden Zugang zu dieser Sammlung. Die im Fließtext verwendeten Verweise auf die Briefe entsprechen dem folgenden System: Ein Buchstabe für den Anfangsbuchstaben der Briefserie entsprechend der Bezeichnung im Archiv, drei Ziffern als Kennzeichnung für die Serie, drei weitere Ziffern verweisen auf den einzelnen Brief in der Sammlung. Der vollständige Datensatz wird online verfügbar sein (https://doi.org/10.6084/m9.figshare.4516772). Eine Visualisierung der geografischen und zeitlichen Zusammensetzung der Sammlung kann dort ebenfalls eingesehen werden.

${ }^{6}$ Die Textstellen werden unkorrigiert wiedergegeben, um enger an der sprachlichen Welt der Briefeschreiber zu bleiben.
} 
für die Empfänger und somit für das Amerikabild in Deutschland, anonymisiert und abstrahiert aber zugleich das Geschriebene etwas von den einzelnen Briefeschreibern.

Die individuellen deutsch-amerikanischen Netzwerke in den USA, die sich aus den Briefen erschließen, umfassen ausgewanderte Familienangehörige, alte und neue deutschsprachige Bekannte sowie Kontakte zu anderen Auswanderern und „Amerikanern“ (ein dehnbarer Begriff, der durchaus früher eingetroffene Migranten und Migrantinnen einschließt). Die Briefe bieten durch die Erwähnung von Treffpunkten und die Beschreibung von Tagesabläufen und Wochenenden Einblicke in die als wichtig erachteten Aspekte deutsch-amerikanischer Netzwerke.

\section{Migrantische Netzwerke, Integration und politische Krisen}

Die folgenden Abschnitte untersuchen drei Krisenepisoden die das transnationale deutsch-amerikanische Kommunikationsfeld strukturieren und das Verständnis der Auswanderer von kollektiver Identität beeinflusst haben. Dabei tritt die kontinuierliche Anpassung dieser Netzwerke zu Tage. In jedem Kommunikationsakt werden die Verbindungen zwischen Individuen neu verhandelt, wodurch bestehende Muster bestärkt oder verändert werden. Die frühen Briefe unterstreichen in diesem Zusammenhang insbesondere die Rolle der sogenannten „starken“, transitiven Beziehungen.

\subsection{Migrantische Netzwerke bis zum Ersten Weltkrieg}

Nach der ersten großen Migrationswelle bildeten sich bis zur Zeit des amerikanischen Bürgerkriegs eine deutschsprachige Infrastruktur in den USA sowie ein rasch expandierendes transatlantisches Kommunikationsfeld heraus. Die Briefe zeigen, dass beide Arten von Netzwerken von Individuen getragen und sich im Kontext geringer staatlicher Intervention weitestgehend ohne äußeren Druck entwickelten. Im 19. Jahrhundert waren die USA besonders offen für diese Art der migrantischen Selbsthilfe, die die schwache staatliche Präsenz in gesellschaftspolitischen Fragen kompensierte (K024_002, K024_006). Ihre soziale Zusammensetzung - Landwirte, Zimmermänner, Brauer aber auch Juristen oder Lehrer - sowie die regionale Konzentration der Auswanderer machten diese deutschsprachigen Netzwerke in den USA von 1850 bis etwa Ende des Jahrhunderts weitgehend autark.

Die Briefe drücken von Anfang an ein starkes Verantwortungsgefühl selbst gegenüber entfernten Verwandten aus, für die teilweise die Kosten der Ausreise vorgestreckt wurden (S038_010). 1868 informiert ein Auswanderer einen seiner Brüder:

Bruder Wilhelm schrieb mir, daß er Lust hätte mir zu folgen und ich freue mich sehr darüber (...). Sei so gut und schreibe du mir umgehend wie es ist, ob er kommen wird, meine Ansicht ist, es wäre besser wenn er erst im Frühjahr die Reise antrete, er muß es aber am besten wissen, wie es mit seinem Militair zustande geht, ich schrieb ihm er sollte mir ganz offen alles mittheilen und wenn ich ihm in irgend einer Weise kann helfen so will ich es ja recht 
herzlich gerne thun, denn ich bin überzeugt daß er hier schneller und besser sein Fortkommen finden wird als draußen, als lediger man, er wird es nicht so schwer finden, als ich es gefunden habe, denn er ist mehr an Arbeit gewöhnt als ich war wie ich nach Amerika kam (B023_015).

In der Frühphase der Auswanderung verließen regelmäßig ganze Familien den Deutschen Bund bzw. ab 1871 das Deutsche Reich. Auf Großfamilien basierende transatlantische migrantische Netzwerke blieben auch bestehen, wenn Briefe über längere Zeiträume unbeantwortet blieben. Das Schreiben und Lesen der Briefe und die dadurch geschaffenen Erwartungen verankerten die transnationalen Netzwerke im migrantischen Alltag. Dadurch wurden die Beziehungen zwischen Individuen und Familien jenseits des eigentlichen Informationsaustausches relevant und schafften eine zeitübergreifende Verbindung, z.B. wenn über Auswanderungspläne oder geteilte Erinnerungen geschrieben wurde.

Die Funktion der persönlichen Netzwerke konnte sich mit jedem neuen Brief verschieben. Berichte von wirtschaftlichen Erfolgen und Misserfolgen oder von Krankheit und Tod konnten eine vorher in Betracht gezogene Auswanderung in Frage stellen oder als besonders dringlich erscheinen lassen. Nach der beschwerlichen Überfahrt kamen Auswanderer häufig bei Verwandten und Bekannten unter und wagten somit aus bestehenden Netzwerken heraus ihre ersten Schritte im neuen Land (S038_010). An dieser Schnittstelle wurde aus den vormals transatlantischen Netzwerken ein neuer Bestandteil der persönlichen deutsch-amerikanischen Netzwerke mit identitätsstiftender Funktion.

Während der Zeit des amerikanischen Bürgerkriegs hatten die deutsch-amerikanischen Netzwerke bereits organisatorische oder rituelle Formen angenommen - es gab deutschsprachige Gemeinden, Schulen, Feierlichkeiten, gemeinsam gelebte Traditionen und Erinnerungen. Sowohl in praktischen als auch in emotionalen Fragen unterstützten diese Netzwerke die Einwanderer - sie dämmten mit der Auswanderung verbundene Risiken ein (W015_058) und schwächten das Gefühl von Heimweh ab (K024_024).

Die Briefeschreiber differenzieren zwischen „alten“, festen Freundschaften oder familiären Verbindungen und den oberflächlicheren Bekanntschaften in „deutschen“ Kreisen (K024_005). Insbesondere die Briefe aus der Heimat unterstrichen, dass die deutsch-amerikanischen Netzwerke kein Ersatz für die wahre Familie in Deutschland sein können:

Du hast Dir viel versucht und bist glücklich durchgekommen daher hoffen und vertrauen wir auch auf Gott, daß er Dich in seinen (...) Schutz nehmen möge, u. erhört er unsere Gebete, damit sehen wir Dich vielleicht bald wieder, denn unser aller herzlicher und einziger Wunsch ist dies, und wenn Du Dir noch etwas wirst versucht haben dann wird auch der Wunsch in Dir rege werden wieder in Dein Vaterlande und zu deinen Blutsverwandten zurück zukehren, denn ob Du gleich dort einige Landsleute und Bekannte hast, so sind dies doch immer keine Blutsfreunde, die es nicht so gut und herzlich mit Dir und Deinem Schicksale nehmen als wir (S038_007). 
Alte persönliche Netzwerke bestanden in den USA fort, und neue wurden bewusst geschaffen, so zum Beispiel durch Heirat innerhalb des deutschen Bekanntenkreises (E009_033) oder durch Patenschaften (E009_050). Familienbedingte Verbindungen, so betonen die Briefe immer wieder, vermittelten ein Gefühl von „Heimat“ (S038_002). Für Einzelpersonen ohne Familienanhang erfüllen die deutschsprachigen Netzwerke in den USA in dieser Phase diese Funktion:

Ich gehe überhaupt gerne zu diesen Leuten, den wenn ich dort bin sprechen wir fast bloß von der Heimath. Man kriegt doch manchmal so ein bischen Heimweh, namendlich wen die Fest und Feiertage kommen, und man muß hir immer fort Arbeiten, ausgenommen einige 4 bis $6 \mathrm{~h}$ welche man frei hat, da tritt der zweite und dritte Feiertag einen so lebhaft vor Augen, das man manchmal glaubt man sei selbst dort, mit meinen Gedanken bin ich auch die ganzen Feiertage bei Euch gewesen (F009_016).

Unser Briefkorpus zeigt, dass Transnationalität umgehend Teil des gelebten migrantischen Alltags wurde. Die fortdauernde emotionale Nähe zu Deutschland war stark familienzentriert und an den Herkunftsort gekoppelt, doch die gleichzeitige Zugehörigkeit zu den USA wurde dadurch nicht in Frage gestellt. In vielen Briefen wird betont, wie viel ,besser“ das Leben in den USA sei; einige unterstreichen ihre Bereitschaft, im amerikanischen Bürgerkrieg zu kämpfen und betonen, dass dort ausschließlich Freiwillige kämpften:

Lieber Schwager und Schwägerin, ihr wolt gerne wissen wie es mit den Kriege hier steht, das habe ich euch schon kurz im vorigen Brief geschrieben, ich wiederhole es nochmals, es wirt keiner gezwungen Kriegsdienst zu thun, es sind jetzt 660.000 freywillige in Dienst, und wen noch so viel verlangt werden, so sind schon wieder so viel bereit, ihr müßt nur betenken, das keiner mehr Gelt machen kan wie der Soltat, der gemeine bekommt 13 Dollar nebst Kost ud Kleitung den Monat und 100 Dollar extra wen der Krieg zu Ente ist, solte einer ums leben kommen, so bekomen seine Angehörigen die Rückständige Monathliche Löhnung nebst 100 Dollar(G012_006).

Viele Einwanderer beschreiben ihre regelmäßigen Begegnungen mit Personen, die schon vor der Emigration zu ihrem Bekanntenkreis zählten (F008_014). Diese Verbindungen verankerten die transnationale Dimension nachhaltig im Leben der Auswanderer, insbesondere an Feiertagen:

$\mathrm{Zu}$ Weihnachten habe ich u. Ernst wieder ein Stelldichein in New York gehabt u. die Feiertage brüderlich miteinander bei meinen alten Lobensteinern verlebt. bei welchen wir völlig einheimisch sind $u$. wie ihre eignen Kinder betrachtet werden, wenn wir gemeinschaftlich einen Besuch bei denselben auf 8 Tage abstatten (R015_002).

Einblicke in das Innenleben der migrantischen Netzwerke unterstreichen in dieser Phase, dass es für die Einwanderer noch keine Selbstverständlichkeit war, sich als Deutsche zu verstehen. Stattdessen werden regionale Identitäten und ihr Zusammentreffen in den USA reflektiert: 
Man fängt jetzt auch an Kirmsen zu feuern, da war ein Schwaben und eine Hessenkirmse da ists lustig hergegangen, Sachsen haben zurück gehalten, weil es hier mehr für einen Skandal als für eine Lustbarkeit gehalten wird. Aber das Weihnachtsfest ist desto mehr gefeiert worden [...] (E009_029).

Ein Zusammengehörigkeitsgefühl als „Deutsche“ entstand entweder im Kontakt mit Einwanderern aus anderen Ländern oder wenn es kollektive Interessenskonflikte gab, so z.B. wenn sich ein Auswanderer in Oskaloosa 1855 darüber beschwerte, dass man vor Ort weder Bier noch Branntwein bekomme und „des Sonntags kein Musick kein Laden darf auf sein“ (B021_011). Familienzentrierte deutsch-amerikanische Netzwerke dienten ebenfalls der finanziellen Absicherung. So konnten bereits ausgewanderte Familienmitglieder Ratschläge beim erstmaligen Erwerb von Eigentum geben:

Wir haben uns jetzt nämlich 2 Häußer gekauft, neben meinem Bruder dieselben habe ich billig erhalten, eins, wo ich drin wohne für 125 Dollars und eins ein Barkhaus für 10 Doll. dieselben stehen auf einer Lot (Hofraum) daselbst habe ich guten Platz für Holz hinzulegen. Den Lot habe ich gerentet auf 10 Jahre, 100 Doll. per Jahr, sehr billig von dem Blockhaus, welches ich ein wenig ausgebessert habe, beziehe ich 21/4 Doll. Rent per Monat, Bruder Heinrich und Emil sind mir dabei mit Rath $\mathrm{u}$. That beigestanden, auch Bruder Christel hat sein Möglichstes gethan [...] Emil hat mir gleich 200 Doll gelehnt welche ich theils in mein Geschäft verwandt habe, theils habe ich die Häuser abbezahlt damit; derselbe hat sich schon viel Geld gespart (E009_052).

Die Familiennetzwerke in den USA blieben auch in Zeiten von Misserfolg ein Rückhalt, der den Adressaten der Briefe deutlich machte, dass in Amerika wirtschaftlicher Erfolg trotz aller Möglichkeiten keine Selbstverständlichkeit war:

Erst hat mein Bruder lange Zeit keine Arbeit für sich können bekommen, er ist überall umher gereist, was hier auch mit viel Geldkosten verknüpft ist, entweder er ist zu bald oder zu spät gekommen, u. nirgends hat es sich wollen passen. Während dieser Zeit sind auch manchmal Krankheiten in seiner Familie vorgekommen. Lore hat 3 Wochen gedient aber dafür keinen cent Lohn bekommen, der Herr hat sie darum betrogen wie es hier vielfältig vorkömmt. [...] Wir müssen sehr eingeschränkt leben, aber deswegen suchen $u$. finden doch auch immer noch andere bei uns Obdach u. Schutz, u. kommen immer Alle gern zu uns, wenn ihn einmal die Arbeit ausgegangen ist, oder die Herrschaften alle geworden sind, wie es immer bei Emma aus Eischleben der Fall ist (E009_062).

Die an der Schnittstelle von transatlantischen und deutsch-amerikanischen Netzwerken geformten Erwartungen stellten durchaus auch eine Belastung dar, der man sich kaum entziehen konnte (B022_014). Zum einen erwarteten Familienmitglieder, dass man sich um kranke Angehörige kümmerte, zum anderen kursierten in den deutsch-amerikanischen Netzwerken dieser Zeit klare Vorstellungen darüber, wen es zu heiraten galt, wobei es zu Spannungen kam wenn derartige Erwartungen enttäuscht wurden und erhoffte Eheschließungen - und damit verbundene Migration 
- nicht stattfanden (F009_002). Eheschließungen waren mit Konflikten verbunden und veränderten allmählich die Netzwerke in den USA - außerhalb der deutschsprachigen Gemeinde zu heiraten bedarf zunächst in den Augen eines Ausgewanderten besonderer Rechtfertigung:

Ich machte die Bekanntschaft mit einem jungen Mädchen, sie und ich dachten, wir konnten beide glücklich leben und so - verheirathete ich mich am 1ten Januar 1866. Seit diesem Tage lieber Bruder kann ich sagen, ich fühle, als hätte ich eine heimath. Meine Frau ist eine geborene Französin, du oder meine anderen Brüder oder Eltern müßt nicht auffallend darüber denken, daß ich zu einem Franzosen übergegangen bin (B023_012).

In der frühen Phase der deutschen Auswanderung bestand eine enge Wechselwirkung zwischen persönlichen transnationalen und deutsch-amerikanischen Netzwerken. Transnationalismus wurde im Alltag gelebt, und „Integration“ bedeutete vor allem, Arbeit zu finden und sich eine neue Lebensgrundlage zu schaffen. Die durch Massenmigration beflügelte deutsch-amerikanische Infrastruktur war ein fester Bestandteil davon, und konnte fehlende Kontakte zu anderen Einwanderergruppen und dem amerikanischen Staat kompensieren.

Die Frühphase der deutschen Auswanderung war somit deutlich von ,starken Beziehungen" im Sinne Granovetters geprägt. Aus der Interaktion familienzentrierter Netzwerke entstanden komplementäre "schwache Beziehungen“ in der Form von personengebundenen und institutionalisierten deutsch-amerikanischen Netzwerken. Krisensituationen wie der Erste Weltkrieg belebten transnationale Netzwerke, da ,authentische“ Informationen über die politische Situation und den Alltag auf beiden Seiten des Atlantiks mit erhöhter Dringlichkeit eingefordert wurden. Daraus spricht die Sorge um Angehörige, aber auch der Wunsch, Unterstützung für Deutschland auszudrücken:

In den Englischen Zeitungen da wird doch nichts wie Lüge rein gesetzt, da gewinne imer die Russen u. Alle, da machen Sie so viele Tausende Gefangene und den andren Tag da wird es in den Deutschen Zeitungen wiederrufen daß es blos Lüge ist. Es wäre wohl zu wünschen daß der Krieg bals Alle wär u. Deutschland würde Siegen. Es wird immer daß beßte von Deutschland geschrieben, blos in den Englischen Zeitungen sein die Deutschen nichts wie Barbaren d (F009_069).

\subsection{Migrantische Netzwerke in der Zwischenkriegszeit}

Am Ende des 19. Jahrhunderts waren deutschsprachige Vereine, Zeitungen und Kirchen ein fester Bestandteil vieler amerikanischer Städte. Zugleich wurde jedoch eine schrittweise Fragmentierung der deutschen Einwanderergesellschaft deutlich. Diese Veränderungen sind Ausdruck der schwächer werdenden institutionellen Verbindungen und sich auflösender deutscher Ballungszentren (S037_011). Die Solidarität mit neuen Einwanderern wurde selektiver (B019_025), und der Ton gegenüber Neuankömmlingen änderte sich mitunter abrupt: 
Das bringen diese kaum eingewanderten, sich als Verwandte brüstenden Anfänger fertig, die Preise so herunterzudrücken, daß keiner mehr einen Dollar verdienen kann, weder sie noch ich. Die Tante in Brooklyn soll ihnen Tausend Dollar zum Anfangen gegeben haben damit wollten sie uns, wie Edwin prahlte, kaput machen (W015_112).

Während des Ersten Weltkriegs, insbesondere nach Kriegseintritt der USA 1917, waren die deutschsprachigen Auswanderer einer anti-deutschen Propaganda ausgesetzt. Auf den ersten Blick verschwanden deutsch-amerikanische Netzwerke in Folge dieses Assimilationsdrucks. Jedoch stellten der Krieg und die anti-deutsche Propaganda auch einen Impuls für die Solidarität mit Deutschland dar. Deutschamerikanische Netzwerke organisierten regelmäßiges Fundraising für Kriegswitwen, -waisen und -versehrte in Deutschland (G011_051) und schickten beachtliche Geldsummen. Auch wenn in der Zwischenkriegszeit weiterhin über die Teilnahme an ,deutschen“ Veranstaltungen berichtet wurde (K025_004), unterstrichen die Briefe, dass derartige Unternehmungen auf besondere Anlässen beschränkt waren (M016_015; M016_048; M016_059; M016_073):

Am Weihnachtstag waren wir dann das erste mal wieder auf einem deutschen Tanz. Ebenso Neujahr, man muß doch schließlich wieder mal unter Menschen, aber geregnet hat es an beiden Tagen (M016_015).

Wir waren heilig Abend wieder bei unseren Bekannten. Wir sind auch Silvester ausgegangen, denn wir 2 allein daheim [trübsal] blasen, das hätt ich nicht aus gehalten, es ging in dem deutschen Lokal auch ganz lustig zu, wir sind erst früh um 5 heim, aber für mich war das nur Galgenhammer (M016_073).

Trotz ihrer schrittweisen Abschwächung erwiesen sich deutsch-amerikanische Netzwerke zunächst als relativ anpassungsfähig. In der Zwischenkriegszeit erfüllten sie einige der für die Frühphase charakteristischen Funktionen. Nachbarschaftliche Verbindungen aus Deutschland konnten zum Teil in den USA fortgeführt werden (B019_028), ebenso wie arbeitsbedingte Netzwerke (S037_005).

Die migrantischen Netzwerke veränderten sich auch dadurch, dass während der Zwischenkriegszeit vermehrt Einzelpersonen und nicht mehr komplette Familienverbände auswanderten. Dadurch verloren die transatlantischen Netzwerke an Verbindlichkeit, und durch die Auflösung der dörflichen Verankerung des Briefverkehrs ersetzte der Austausch zwischen Einzelpersonen die Kommunikation zwischen größeren Gruppen. Auch wenn sich die Zusammensetzung transnationaler Netzwerke veränderte, blieben diese Verbindungen für Auswanderer durchaus wichtig:

Nun 1. M. wie Du siehst bin ich schon in den Staaten. Geht mir gut hier, habe auch den zweiten Tag gleich Arbeit gefunden. In der Wohnung bin ich beim Fischerbaum Sepp, Stäl Karl ist auch bei mir. Die ganzen Neukirchner hab ich schon getroffen, nur den Lechner Anton nicht. Köppelle Sepp hat mich schon rasiert. Letzten Namenstag war der Haiwier oder Haimler Kirta, mein 1. Marichen, da hat eschte bairische Knödeln gegeben, Küchl und ein gutes Bier (B019_028). 
Insbesondere Verwandte boten einen Rückhalt, auch wenn es in den Briefen nicht mehr so häufig betont wurde:

Also mir gefällt es sehr gut in New York, die Leute für die ich schaff sind so nett, u. alle die Verwandten sind ganz verrückt nach mir, und wissen nicht, was sie tun sollen, damit mirs ja gefällt $\mathrm{u}$. ich heim [fürchte], du glaubst garnicht, wie leicht alles hir ist (M016_009).

In der Zwischenkriegszeit arbeiteten Auswanderer bereits seltener für Verwandte oder Bekannte. Die wirtschaftliche Funktion der Netzwerke war somit eine indirektere, obwohl Arbeitsvermittlung insbesondere für die ersten beruflichen Schritte weiterhin bedeutend sein konnte (S037_018):

Wenn Du Ib. Mary die Bürgerpapiere nun von jemanden bekommen hast \& nach Winona kommen magst bist Du natürlich herzlich willkommen, denn wenn Du hier bist kannst Du alles bei uns haben - wo dein Herz sich sehnt in fremden Lande die erste Zeit - Heimat! Wir werden dich gerne aufnehmen $\&$ in allem Deine Heimat ersetzen, wir beide werden wieder alte Freunde sein wie in der Ib. alten Heimat. [...] Stellungen gibt es viele hier. Auch deutsche Familien wo Du lernen kannst.

Im Zuge des Ersten Weltkriegs gerieten die bereits im Abbau begriffenen ,schwächeren" Beziehungen der deutsch-amerikanischen Netzwerke weiter unter Druck, während ,starke“ transatlantische Netzwerke durch die politische Konfrontation zwischen Deutschland und den USA teilweise neu belebt wurden.

\subsection{Migrantische Netzwerke nach dem Zweiten Weltkrieg}

Nach dem Zweiten Weltkrieg änderten sich der Charakter und die Funktion der migrantischen Netzwerke grundlegend. ${ }^{7}$ Transatlantische und deutsch-amerikanische Netzwerke blieben zwar ansatzweise bestehen, doch ihre wirtschaftliche Funktion lässt sich in den Briefen kaum mehr ausmachen, ebenso wenig wie eine separate deutsch-amerikanische Identität.

Insbesondere an Festtagen dienten deutsch-amerikanische Kreise dazu, ein gewisses deutsches Brauchtum aufrecht zu erhalten. Diese Netzwerke bestanden aus Familienmitgliedern und Freunden, die nun nicht mehr in unmittelbarer Nähe zueinander wohnten (M016_122). Auch wenn diese Netzwerke seltener aktiviert wurden, erwähnten die Einwanderer solche Treffen in ihren Briefen, wahrscheinlich auch weil sie davon ausgehen konnten, dass sie für die Empfänger eine besondere Bedeutung hatten.

Als Orientierungshilfe waren familienzentrierte Netzwerke nach dem Zweiten Weltkrieg von geringerer Bedeutung (M016_134). Auswanderer in der dritten Generation assoziierten deutsch-amerikanische Netzwerke kaum mehr mit ihrer Zukunftsplanung. Deutschland wurde selbst zu einer Erinnerung, die schrittweise romantisiert und bei gemeinsamen Familientreffen aktualisiert wurde. Durch die deutsch-ameri-

\footnotetext{
${ }^{7}$ Die Zeit des Zweiten Weltkriegs und die Nachkriegszeit sind in der Briefesammlung nur punktuell abgebildet.
} 
kanischen Netzwerke wurde ein Bild von Deutschland aufrechterhalten, auch wenn dies für den Alltag der Auswanderer keine einflussreiche Rolle mehr spielte. Insofern sie noch bestand, war eine kollektive deutsche oder deutsch-amerikanische Identität vom politischen Geschehen losgelöst (W015_077).

\section{Zusammenfassung}

Unser Einblick in die transatlantischen und deutsch-amerikanischen migrantischen Netzwerke gibt Aufschluss über die zentrale Rolle der über einen langen Zeitraum hinweg bestehenden ,starken“ familienzentrierten transnationalen Beziehungen und zeigt, wie sich die Funktionen dieser Netzwerke mehrmals veränderten. Im 19. Jahrhundert waren sie als Hauptinformationsquelle für potenzielle Auswanderer eine Voraussetzung für die Massenemigration. Zugleich beschreiben die Briefe die regelmäßigen Beziehungen zu Verwandten, neuen und alten deutschen Bekannten sowie die Teilhabe an einem institutionalisierten ,deutschen“ Netzwerk aus Vereinen, Kirchen und Brauereien, einem ,deutschen“ Arbeitsmarkt und „,deutschen“ Nachbarschaften. Die deutsch-amerikanischen Netzwerke speisten sich direkt aus den „starken“ transnationalen Netzwerken - so wurden starke und schwache Netzwerke miteinander verknüpft. Die deutsch-amerikanischen Netzwerke entwickelten dann eine Eigendynamik, die die zum Teil erheblichen Pausen zwischen den Briefen in die alte Heimat überbrückte. Die Briefe veranschaulichen, wie deutsche Traditionen bewusster gelebt wurden und somit ein wichtiger Teil der Identitätsentwicklung der Migranten und Migrantinnen waren. Die vielen Bezüge auf die deutsche Sprache, deutsche Traditionen und ausgeprägte deutsch-amerikanische Netzwerke für die alltäglichen Belange werden im Gegenzug die Wahrnehmung der Empfänger der Briefe geprägt haben.

Im 20. Jahrhundert konzentrierten sich die in den Briefen widerspiegelnden transnationalen Beziehungen stärker auf die Familie im engeren Sinne sowie auf weniger verbindliche deutsch-amerikanische Netzwerke. Durch die interne Diversität und Mobilität der deutschen Auswanderer sowie den wachsenden Assimilationsdruck in den USA wurden die deutsch-amerikanischen Netzwerke schwächer, während transnationale, familienzentrierte Beziehungen wiederbelebt wurden. Die Ausgewanderten fragten nun zunehmend Informationen aus der Heimat an - diese Tendenz zeigt sich verstärkt in Krisen- und Kriegszeiten. In der Entwicklung von einem primär ländlichen Arbeitsmarkt hin zu einer fortschreitenden Beschäftigung in Industrie und Handel dienten bestehende Netzwerke nun eher dazu, Informationen über Arbeit auszutauschen, aber keine Beschäftigung an sich zu erlangen. Der Zweite Weltkrieg markierte eine weitere Zäsur - transnationale und deutsch-amerikanische Netzwerke existierten nur noch punktuell und erhielten sich durch wechselseitige Bezüge in den Briefen.

Die longue durée Perspektive der Auswandererbriefe zeigt, wie transatlantische und deutsch-amerikanische Netzwerke ineinander verschränkt sind, sich gegenseitig bedingen und je nach Zeitabschnitt und politischem Kontext ihre jeweilige Bedeutung im migrantischen Alltag variiert. Unsere historische Fallstudie unterstreicht, dass transnationale Familiennetzwerke sich nicht nur parallel zu Integrationsprozes- 
sen entwickeln, sondern diese aktiv unterstützen können. Dadurch wird deutlich, dass Integration ins Einwanderungsland ein vielschichtiger Prozess ist, bei dem die kulturelle Anbindung ans Heimatland parallel zur wirtschaftlichen und gesellschaftlichen Integration existiert. Die Fokussierung auf die Zusammensetzung, Kontinuitäten und Verschiebungen in den migrantischen Netzwerken anhand der Briefe erweitert somit den Horizont bisheriger historischer räumlich und zeitlich begrenzter Studien. Dadurch tritt die Gleichzeitigkeit transnationaler und (deutsch-)amerikanischer Bezüge noch stärker ins Blickfeld.

Open Access Dieser Artikel wird unter der Creative Commons Namensnennung 4.0 International Lizenz (http://creativecommons.org/licenses/by/4.0/deed.de) veröffentlicht, welche die Nutzung, Vervielfältigung, Bearbeitung, Verbreitung und Wiedergabe in jeglichem Medium und Format erlaubt, sofern Sie den/die ursprünglichen Autor(en) und die Quelle ordnungsgemäß nennen, einen Link zur Creative Commons Lizenz beifügen und angeben, ob Änderungen vorgenommen wurden.

\section{Literatur}

Ahmadov, Anar, und Gwendolyn Sasse. 2015. Migrants' regional allegiances in homeland elections: evidence on voting by poles and ukrainians. Journal of Ethnic and Migration Studies 41(11):1769-1793.

Attebery, Jennifer. 2007. Up in the rocky mountains: writing the Swedish immigrant experience. Minneapolis: University of Minnesota Press.

Bade, Klaus J. 2003. Migration in European history. Malden: Blackwell Pub.

Bergquist, James. 1984. German communities in American-cities: an interpretation of the $19^{\text {th }}$ century experience. Journal of American Ethnic History 4(1):9-30.

Bommes, Michael. 2011. Migrantennetzwerke in der funktional differenzierten Gesellschaft. In Netzwerke in der funktional differenzierten Gesellschaft, Hrsg. M. Bommes, V. Tacke, 241-259. Wiesbaden: VS.

Boyd, Monica. 1989. Family and personal networks in international migration - recent developments and new agendas. International Migration Review 23(3):638-670.

Bulmer, Martin. 1983. 'The Polish peasant in Europe and America': a neglected classic. Journal of Ethnic and Migration Studies 10(3):470-476.

Burgess, Katrina. 2012. Collective remittances and migrant-state collaboration in Mexico and El Salvador. Latin American Politics and Society 54(4):119-146.

Conzen, Kathleen N. 1980. Germans. In Harvard encyclopedia of American ethnic groups, Hrsg. S. Thernstrom, 405-425. Cambridge: Belknap Press.

Elrick, Tim, und Emilia Lewandowska. 2008. Matching and making labour demand and supply: agents in Polish migrant networks of domestic elderly care in Germany and Italy. Journal of Ethnic and Migration Studies 34(5):717-734.

Fairchild, Henry. 1913. Immigration a world movement and its American significance. New York: Macmillan Co.

Freeman, Gary. 2004. Immigrant incorporation in western democracies. International Migration Review 38(3):945-969.

Gans, Herbert. 1962. The urban villagers: group and class in the life of Italian-americans. New York: Free Press of Glencoe.

Glick Schiller, Nina, und Georges Fouron. 1999. Terrains of blood and nation: Haitian transnational social fields. Ethnic and Racial Studies 22(2):340-366.

Grabbe, Hans-Juergen. 1997. Weary of Germany - weary of America. Perceptions of the United States in nineteenth-century Germany. In Transatlantic images and perceptions: Germany and America since 1776, Hrsg. D.E. Barclay, E. Glaser-Schmidt, 65-86. Cambridge: Cambridge University Press.

Granovetter, Mark. 1973. The strength of weak ties. American Journal of Sociology 78(6):1360-1380.

Green, Nancy, und Roger Waldinger. 2016. Introduction. In A century of transnationalism: immigrants and their homeland connections, Hrsg. N. Green, R. Waldinger, 1-31. Urbana: University of Illinois Press.

Guillet, Edwin. 1963. The great migration: the Atlantic crossing by sailing-ship since 1770. Toronto: University of Toronto Press. 
Haug, Sonja. 2008. Migration networks and migration decision-making. Journal of Ethnic and Migration Studies 34(4):585-605.

Hawgood, John. 1940. The tragedy of German-America: the Germans in the United States of America during the nineteenth century - and after. New York: G.P. Putnam's Sons.

Helbich, Wolfgang. 1988. „Alle Menschen sind dort gleich... “ Die deutsche Amerika-Auswanderung im 19. und 20. Jahrhundert. Düsseldorf: Schwann.

Herz, Andreas, und Claudia Olivier. 2012. Transnational social networks - current perspectives. Transnational Social Review 2(2):115-119.

Immigration Commission, und William Dillingham. 1911. Reports of the immigration commission. Washington: Government Printing Office.

Kazal, Russell. 2004. Becoming old stock: the paradox of German-American identity. Princeton: Princeton University Press.

Kellor, Frances. 1920. Immigration and the future. New York: George H. Doran.

Kornblum, William. 1974. Blue collar community. Chicago: University of Chicago Press.

Krabbendam, Hans. 2009. Freedom on the horizon: Dutch immigration to America, 1840-1940. Grand Rapids: William B. Eerdmans Pub. Co..

Lacroix, Thomas. 2009. Transnationalism and development: the example of Moroccan migrant networks. Journal of Ethnic and Migration Studies 35(10):1665-1678.

Levitt, Peggy. 2001. The transnational villagers. Berkeley: University of California Press.

Levitt, Peggy, und Glick Nina Schiller. 2004. Conceptualizing simultuneity: a transnational social field perspective on society. International Migration Review 38(3):1002-1039.

Liu, Haiming. 2005. The transnational history of a Chinese family: immigrant letters, family business, and reverse migration. New Brunswick: Rutgers University Press.

Liu, Mao-Mei. 2013. Migrant networks and international migration: testing weak ties. Demography 50(4):1243-1277.

Massey, Douglas, und Felipe Espana. 1987. The social-process of international migration. Science 237(4816):733-738.

Mikoletzky, Juliane. 1988. Die deutsche Amerika-Auswanderung des 19. Jahrhunderts in der zeitgenössischen fiktionalen Literatur. Tübingen: M. Niemeyer.

Miller, Kerby. 2008. Ireland and Irish America: culture, class, and transatlantic migration. Dublin: University of Notre Dame.

Poros, Maritsa. 2001. The role of migrant networks in linking local labour markets: the case of Asian Indian migration to New York and London. Global Networks 1(3):243-260.

Roeber, Gregg. 1997. "Through a glass, darkly". Changing German ideas of American Freedom, 1776-1806. In Transatlantic images and perceptions: Germany and America since 1776, Hrsg. D.E. Barclay, E. Glaser-Schmidt, 19-40. Cambridge: Cambridge University Press.

Serra, Ilaria. 2009. The imagined immigrant: images of Italian emigration to the United States between 1890 and 1924. Madison: Fairleigh Dickinson University Press.

Smith, Robert. 2000. How durable and new is transnational life?: historical retrieval through local comparison. Diaspora: A Journal of Transnational Studies 9(2):203-233.

Taylor, Philip. 1971. The distant magnet: European emigration to the U.S.A. London: Eyre and Spottiswoode.

Thomas, William Isaac, und Florian Znaniecki. 1927. The Polish peasant in Europe and America. Boston: Gorham Press.

Wimmer, Andreas, und Glick Nina Schiller. 2002. Methodological nationalism and beyond: nation-state building, migration and the social sciences. Global Networks 2(4):301-334. 